\title{
Effects of a whole body compression garment on recovery after a heavy resistance
}

\author{
Ahmad ARBABI \\ Department of Physical Education and Sport Science, University of Zabol, Zabol, Iran \\ Address correspondence to A. Arbabi, ahmadarbabi@uoz.ac.ir
}

\begin{abstract}
This study aimed to investigate the effects of wearing a whole body compression garment on recovery from a typical heavy resistance training workout in resistance trained men and women. Fourteen men (mean \pm SD: age, $23.0 \pm 2.9$ years) and fourteen women (mean \pm SD: age $23.1 \pm 2.2$ years) who were highly resistance trained gave informed consent to participate in the study. A within-group (each subject acted as their own control), balanced, and randomized treatment design was used. Nutritional intakes, activity, and behavioral patterns (e.g., no pain medications, ice, or long showers over the 24 hours) were replicated 2 days before each test separated by 72 hours. An 6-exercise whole body heavy resistance exercise protocol using barbells (10 repetition of 3 sets at $70 \%$ of one- repetition maximum, 2.0 minute rest) was performed after which the subject showered and put on a specific whole body compression garment one designed for women and one for men (CG) or just wore his/her normal noncompression clothing (CON). Subjects were then tested after 24 hours. Dependent measures included sleep quality, vitality rating, resting fatigue rating, muscle soreness, muscle swelling via ultrasound, reaction movement times, bench throw power, countermovement vertical jump power, and serum concentrations of creatine kinase (CK) measured from a blood sample obtained via venipuncture of an arm vein. We observed significant $(\mathrm{p} \leq 0.05)$ differences between $C G$ and $C O N$ conditions in both men and women for vitality (CG, CON), resting fatigue ratings (CG, CON), muscle soreness (CG, CON), ultrasound measure swelling (CG , CON), bench press throw (CG .CON), and CK (CG, CON). A whole body compression garment worn during the 24-hour recovery period after an intense heavy resistance training workout enhances various psychological, physiological, and a few performance markers of recovery compared with noncompressive control garment conditions. Wearing a CG appears to help in the recovery process after intense heavy resistance training in men and women.
\end{abstract}

Keywords: Compression garment, muscle damage, recovery, resistance, soreness.

\section{INTRODUCTION}

Because athletes commonly perform intensive physical training or competitions on consecutive days, rapid recovery of performance is important to maximize competitive success and prevent excessive fatigue (1). Several strategies are used in sports to aid the recovery process, including massage, active recovery, water immersion, contrast bathing, and macronutrient supplementation $(2,14)$. Each exercise protocol or sport competition can result in various magnitudes of alterations in physiological or psychological or both alterations (e.g., increased soft tissue damage, increased fatigue states, increased core temperature, and so on) $(12,40)$. The rapidity of recovery can be influenced by many factors including genetics, diet, fitness level, sleep, environment, winning or losing, and the magnitude of the physical/psychological trauma. Thus, the exercise demands and the recovery processes are specifically related to the stressors the athlete or individual is exposed to. In sports, the use of CG during competition is hypothesized to delay the onset of fatigue or increase power output. Although some experimental studies have demonstrated potential beneficial effects of wearing a CG during exercise (38), several previous investigations did not confirm the performance-enhancing effect of the use of a CG during exercise $(4,11,28)$. Jakeman et al. (15) compared the jump performance recovery after 100 plyometric drop jumps in female volunteers between those who used a CG for $12 \mathrm{~h}$ after exercise and those who did not. Subjects who wore the CG showed significantly faster recovery of jump performance. The use of a CG for $24 \mathrm{~h}$ after repeated $20-\mathrm{m}$ sprints and 10 plyometric bounds resulted in significantly lower muscle soreness $24 \mathrm{~h}$ after the exercise than that in the control group (8). Customized garments for various specific functions are becoming more popular (e.g., swimmers wear a specific compressive garment for improving their speed in the water). Each garment construction 
potentially interacts differentially with the psychological and biological interfaces with the human and his/her various recovery variables. The lack of effects typically revolves around the mismatch of the garment to the desired function (e.g., low compression and proprioception) or compensatory effects by other endogenous (e.g., anabolic hormone signaling) or exogenous (e.g., drug therapy) operational systems. Several basic mechanisms mediate a garment's capability to achieve any measurable positive benefit. The first mechanism that allows for optimal use is proper fit (17). With high compression or with compression used to treat extreme soft tissue injury, compression may augment feelings of discomfort during the period the garment is used despite the positive results because of mechanical blocking of edema (39). Nevertheless, proper fit (e.g., seams not problematic or constrictive and compression relief in areas of high sensitivity) and feel (e.g., garment material) are the first mechanisms that set the stage for its effects especially with long-term wear. Compression (e.g., 24-27\%) and construction create the potential for optimal skin contact, which is vital for proprioception (kinesthetic sense), which mediates many of the garments performance effects (36). It is vital that garment movement is minimal and stays in contact with the skin, so no air bubbles are produced breaking the special linkage with the stimulation of skin receptors. Optimal compression also allows the body structures to be held in place reducing the amount of movement and oscillation upon contact or allows limbs to be kept in anatomical positions when not used allowing straight-line tissue repair geometrics $(19,20)$. Compression has also been linked to enhancing the "muscle pump" allowing blood return to the heart for removal of waste products $(3,27)$. Finally, mechanical support is needed when added force is required for a movement, and this seems possible only with high compression and material mechanics not optimal for anything but short-term use (39). Considering that most trained athletes often conduct daily training or compete several times in the same day, we wondered how many hours are necessary to promote recovery by wearing a CG in the postexercise period Thus, the purpose of this investigation was to examine the effects of wearing a whole body compression garment after a whole body heavy resistance training workout on psychological and physiological and performance variables after 24 hours of recovery.

\section{MATERIAL \& METHODS}

\section{Subjects}

Each subject had the experimental risks and of the study explained to them and subsequently gave written informed consent to participate. The subjects used in this investigation were considered resistance trained each participating in a their own periodized heavy resistance training program for at least the past 2 years, which included the type of protocol used in the study. The subject's characteristics were as follows: Men $(\mathrm{n}=14)$ (mean $\pm \mathrm{SD}$ : age [years] $24 \pm 3.1$, height $[\mathrm{cm}] 177 \pm 8.5$, body mass $[\mathrm{kg}] 83.2 \pm 8.9$ ) and trained women $(\mathrm{n}=14)$ (mean \pm SD: age [years] 23.6 \pm 2.7 , height [cm] $162.3 \pm 6.4$, body mass [kg]

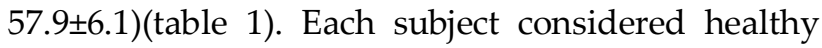
was medically screened by a physician as having no medical conditions that would confound any of the experimental variables. A registered dietician also screened each subject for dietary habits and instructed them on replicating diet profiles for the 2 days before the study and during the 24-hour recovery period. Each subject was instructed on proper water behavior to ensure normal hydration status for all testing because hypohydration has been shown to impact physiological responses and performance (16). Each subject had their garment properly fitted, assuring that the compression levels were appropriate and that garment fit properly per the sizing requirements.

\section{Procedures}

Diet and activity profiles were maintained for 48 hours before the experimental resistance exercise protocol day. Food and beverage choices were standardized throughout the study. The diet repetition protocols consisted of a 48-hour period, which began 24 hours before exercise session and lasted until 12 hours before recovery testing session, which marked the beginning of a 12- hour fasting period. Additionally, subjects were instructed to abstain from exercise outside of the study and drinking more than 2 cups or servings of caffeinated or alcoholic beverages during dietary control period. Subjects were instructed to continue any supplement regimens (e.g., vitamins and so on) in use before study and to not discontinue or begin consumption of any new supplement during the study. Subjects met with registered dieticians to ensure that standardized dietary practices did not provide reason to suspect adverse effects on recovery capability (i.e., insufficient caloric consumption). Subjects replicated all behaviors for each testing phase from use of activity and nutrition. Design 
used a balanced, randomized, within-group treatment. Each subject dressed in typical workout gear, performed the workout, and then showered and changed into a whole body compression garment or noncompressive clothing for the 24 hours of recovery. Subjects removed their recovery garments and then performed all of the testing. Compression Garments and Recovery reduce variance in experimental measures. All experimental tests were practiced and pilot tested before beginning experimental data collection. To minimize variance associated with intertester variability, each experimental procedure was administered by same research team. The $8-10$ repetition maximum (RM) load for each exercise used in the workout was determined during the familiarization phase of the study under the context of performing the total workout as previously described for performing total workout sequence loading (26). In addition, subjects were trained with the protocol and loads for several workouts during the familiarization phase to reduce the novelty and assure similar physiological responses each day.

\section{Resistance Exercise Protocol}

The subjects visited the laboratory to perform the heavy resistance exercise protocol. This protocol was chosen because it would produce a dramatic whole body demands metabolically and stimulate a large amount of muscle mass and create a typical workout challenge in men and women. Upon entry into laboratory, it was confirmed that subjects adhered to experimental controls (i.e., no medications, alcohol, hydrotherapy, long showers, and so on), a 12-hour fasting state, hydration status normal, standardized diet, and had not exercised (24). The 2 series of exercise/ recovery sessions were typically completed per week, with at least 72 hours separating each exercise session. Because all subjects acted as their own control, each subject was scheduled at the same time in the morning to perform the resistance exercise protocol. Upon arrival at the exercise facility, subjects completed a standardized warm-up, consisting of 10 minutes of stationary cycle ergometer pedaling at 50-60 rpm, immediately followed by various dynamic stretching exercises. Following warm-up, subjects completed a standardized free weight resistance exercise protocol incorporating the following 6 exercises (in order): 1) Back squats 2) Bench press 3) Romanian dead lift 4) Biceps curl 5) Sit-up 6) High pull from a hang. Each exercise incorporated 3 sets using the 8-10 RM zone load. Two minutes of rest were given between sets and exercises. Subjects were encouraged to drink water ad libitum. Upon completion of the exercise protocol, subjects were escorted to a locker room, where they showered and changed into either their normal garments without compression or the whole body compression garment. Subject showered and changed into the garment or noncompressive cloths within 20 minutes of the exercise protocol. The compression suits were male- or female-specific whole body compression suits constructed to produce needed compression and allow longterm wear with comfort (75\% nylon and 25\% spandex.

\section{Recovery Testing}

\section{Blood Sample}

A blood sample was obtained via sterile technique from the antecubital vein using a 20-gauge needle and vacutainer. Blood samples were obtained under resting and 12-hour fasted conditions with all subjects sitting rested for 10 minutes before draw time. Blood was centrifuged at 3,000 rpm and 4_C, plasma was aliquoted into specimen, and tubes were stored at 280_C before analyses. Upon completion of blood draw, subjects were escorted to performance testing laboratory and were given a standard breakfast bar (Advocare International, Carrollton, TX, USA) to eat and to drink water before the remaining testing procedures. Plasma was analyzed in duplicate for creatine kinase $(\mathrm{CK})$, lactate dehydrogenase (LDH), and clinical chemistries using standard automated clinical assays by a reference laboratory.

\section{Psychological Questionnaires and Scales}

While standing, subjects were palpated over various muscle groups and rated their perceived soreness on a likert scale of 0 (none) to 10 (maximum possible) for each respective palpation. Subjects then sat and completed 3 perceived muscle soreness/pain scales: one likert 5-point soreness scale for different body parts and a perceived soreness/pain was measured using a visual analog scale (VAS) consisting of a $10-\mathrm{cm}$ line labeled from left (no soreness) to right (extreme soreness) (Kraemer et al., 2009). Subjects also completed one 10-point general fatigue scale, one Profile of Mood States scale, a 6point vitality scale, and one sleep scale/log (Buysse et al., 2000; Faude et al., 2009). Subjects were given as much time as needed to complete the scales, as needed, and external disturbances and distracters were limited.

\section{Ultrasound Analyses}


Using Acuson 13.0-MHz linear array transducer and an Aspen cardiac ultrasound imaging system (Acuson, Elmwood Park, NJ, USA) vastus lateralis swelling thickness and distal patellar tendon thickness were measured per previously referenced techniques (25,30,31). Accuracy and precision of measurements were optimized by the use of permanent marker for exact replication of measurement sites throughout the study. Anatomical references were also used to ensure precision of measurement sites. The same technician conducted all tests throughout the study. The technician administering ultrasound, making the measurements, and analyzing the results was not aware of the specific garments worn by each subject during the recovery period.

\section{Circumference Measurements}

To assess edema resulting from strenuous resistance exercise, circumference measurements of various muscle belly regions were obtained per previously described methods (24). Measurement points were marked with a permanent marker at the beginning of the study and remarked throughout the study to ensure precision of measuring sites. Circumference measures of the upper arm, forearm, upper leg, lower leg, and ankle were taken using a spring-loaded flexible tape measure. The dominant arm and leg were used for all measurements, and all measuring points represented the areas of greatest circumference on each respective limb area.

\section{Bench Throw and Squat Jump}

The bench throw and squat jump performance tests on a laboratory were conducted to assess the effects of each compression garment on peak upperand lower-body power production. Force, power, and velocity were assessed using an AMTI force plate (Advanced Mechanical Technology, Inc., Watertown, MA, USA) and linear transducer in combination with a Myotest wireless accelerometer (Myotest Inc., Royal Oak, MI, USA) that was placed on the bar being lifted. Load was based on body mass at the familiarization visit and kept constant throughout the study to remove variance of findings as a result of different loading schemes. Resistances were set at $20 \%$ of body weight on the bench throw and $50 \%$ of body weight on the squat jump. Subjects completed 3 separate repetitions (assumed fully extended or standing position before beginning each repetition) in each of 2 sets. Subjects were given as much time as needed before feeling ready to begin second set. Subjects were given standard verbal reinforcement throughout attempts. Upon completion ofeach set, peak force, peak velocity, and peak power measures were recorded with the best scores used for analyses.

\section{Countermovement Vertical Jump}

After warming up (using exercise protocol), a 10-repetition countermovement jump was conducted to assess peak power, average power, and maximum performance decrement in lowerbody jumping. A Tendo Linear Transducer cord was attached to a belt-like waist wrap, over the spine. Subjects stood over the top of the transducer, facing away with feet shoulder width apart and hands placed on hips. Subjects were instructed to exert maximum effort on every jump and not pace their jumps. Ten jumps were completed in a continuous fashion under a self-controlled cadence with no breaks between repetitions.

\section{Movement-Reaction Time}

A novel test was used to determine the composite movement-reaction time using a ruler dropquick pinch method. While standing with the lower aspect of dominant arm supported on the marked corner of a standardized table top with a neutral hand position, the tester positioned a meter stick directly above the top of the thumb and index finger in an open prepinch position (approximately 1-inch gap between fingers). Unannounced, the tester would then drop the ruler with the subject pinching and stopping ruler as quickly as possible. The distance the ruler fell between thumb and index finger before being stopped was recorded at the top of the thumb. Three trials were conducted with the best score used for analysis.

\section{Statistical Analyses}

These data are presented as means \pm SD. An independent t-test was used to compare demographic characteristics between men and women. The statistical evaluation of the experimental data was accomplished using a 2-way analysis of variance for treatment condition and sex. When appropriate, Fisher's LSD post hoc tests were used to determine pairwise differences. A $\mathrm{p} \leq 0.05$ was defined as being statistically significant.

\section{RESULTS}

The results of this study demonstrated that in both men and women, compressive garments influence positive effects on recovery in various physiological and performance profiles.

The 5-point likert scale ratings showed no differences in limb and body part soreness ratings 
but a significantly lower rating for the composite torso ratings for the right and left sides of the body in both men and women (men: $0.60 \pm 0.3$ garment vs. $1.8 \pm 0.5$ control and women: $0.61 \pm 0.3$ garment vs. $0.99 \pm 0.4$ control).

On the 10-point generalized muscle soreness scale, ratings were significantly lower in both men and women with the use of the compression garments. Men experienced significantly higher soreness levels than women in the noncompression condition. No differences were observed for the compression garment conditions (Figures 1 and 2). This was replicated in the general soreness VAS scale ratings.

The amount of swelling determined via ultrasound techniques was significantly lower in the thigh in the compression garments vs. that in the control conditions. No sex differences were observed (Figures 1 and 2). No significant differences were observed in the patella thickness values under either condition but men had significantly higher values. No significant differences in limb circumferences were observed.

The bench throw demonstrated a significantly higher performance level with the use of the compression garments compared with the control conditions (Figures 1 and 2). Men showed the expected significantly higher values compared with women.

No significant differences were observed between the treatment conditions for the reaction/movement times. No significant differences were observed for the squat jump, albeit expected sex differences existed for force and power outputs.

\begin{tabular}{lcc} 
Table 1. Subjects characteristics. & \\
\hline & Men & Women \\
\hline & & \\
Age (years) & $24 \pm 3.1$ & $23.6 \pm 2.7$ \\
Height $(\mathrm{cm})$ & $177.3 \pm 8.5$ & $162.3 \pm 6.4$ \\
Body mass $(\mathrm{kg})$ & $83.2 \pm 8.9$ & $57.9 \pm 6.1$ \\
& & \\
\hline
\end{tabular}

No significant differences were observed for the countermovement vertical jump for peak power, average power, and maximum performance decrement between conditions, but again expected sex differences were observed in power outputs.
Fatigue ratings were significantly lower in the compression garments than under control conditions in both men and women, and no sex effects were noted (Figures 1 and 2). No differences were observed in any of the other mood states between treatment conditions. Sleep ratings with 0 meaning very light sleep and 10 meaning very good sleep quality were not significantly different between conditions (women: garment, 7.3 \pm 2.4 vs. control, $7.8 \pm 2.1$ and men: garment, $5.3 \pm 2.1$ vs. control, 5.7 \pm 2.6$)$, although men showed significantly lower quality of sleep for both conditions compared with women.

Vitality ratings were significantly higher with use of the compression garment compared with those under the control conditions (Figures 1 and 2), and no sex effects were noted.

Creatine kinase values were significantly lower at rest 24 hours after the workout after wearing the garment compared with the control conditions for both men and women. Men also showed significantly higher values under both conditions (Figures 1 and 2). Lactate dehydrogenase values reflected this same response pattern. As expected with the nutritional controls, all the values for clinical chemistries (electrolytes, proteins, and so on) were in normal ranges and not significantly different between treatments or were any sex differences noted.

\section{DISCUSSION}

The main finding of the present study is that wearing a CG after heavy resistance exercise did produce more rapid recovery of selected psychological, perceptual, physiological, and performance variables when compared with the use of a noncompression treatment condition over a 24hour recovery time frame. However, not all parameters were affected The resistance exercise protocol incorporated whole body exercises, with relatively high volume, and short-moderate rest periods to produce significant neuromuscular recruitment, metabolic and hormonal responses $(21,22)$. We used a 24 -hour recovery period because many athletes or highly active individuals want to be ready to train or compete within this time frame (12). 

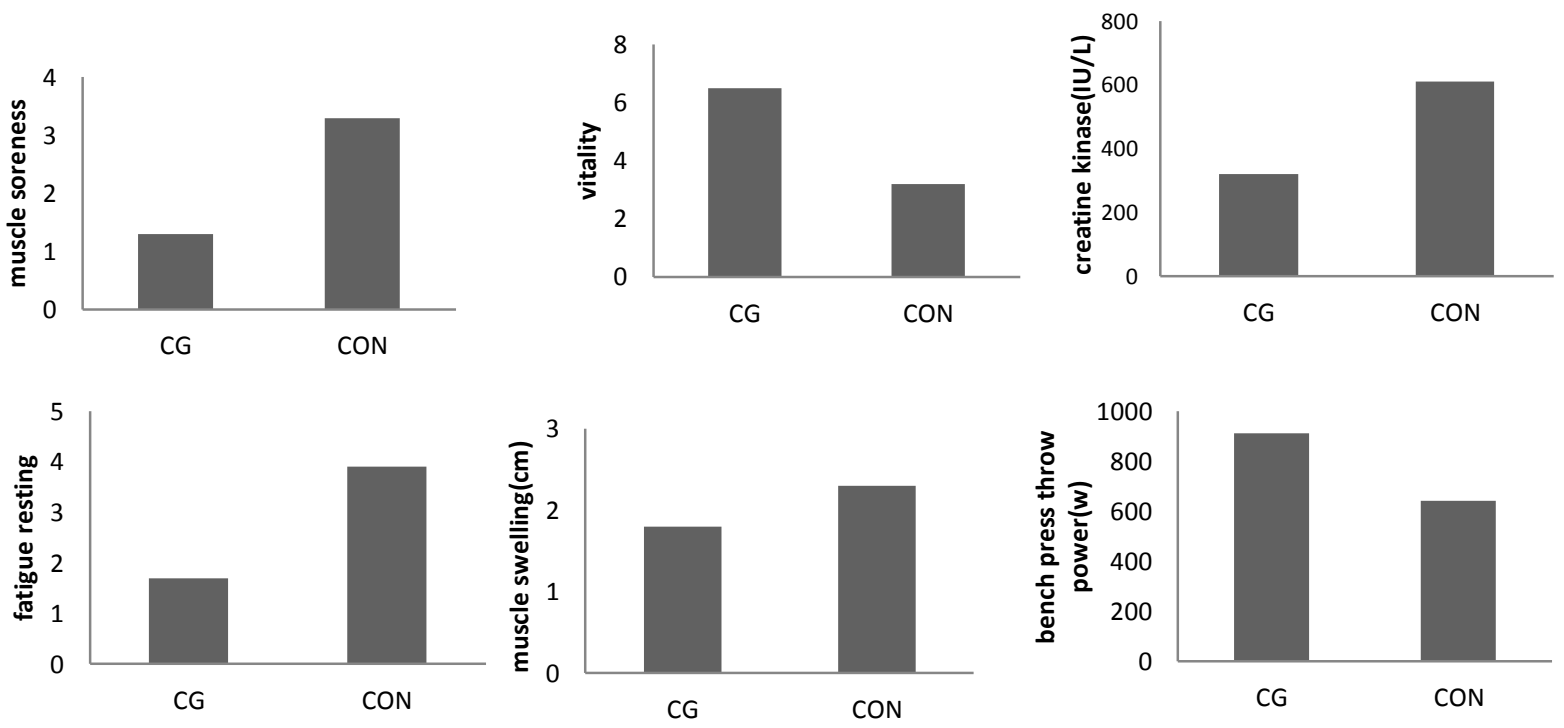

Figure1. The mean $\pm \mathrm{SD}$ of the various parameters are presented for the men comparing the compression garment(CG) with the control conditions(CON).p $\leq 0.05$ from corresponding CG condition.
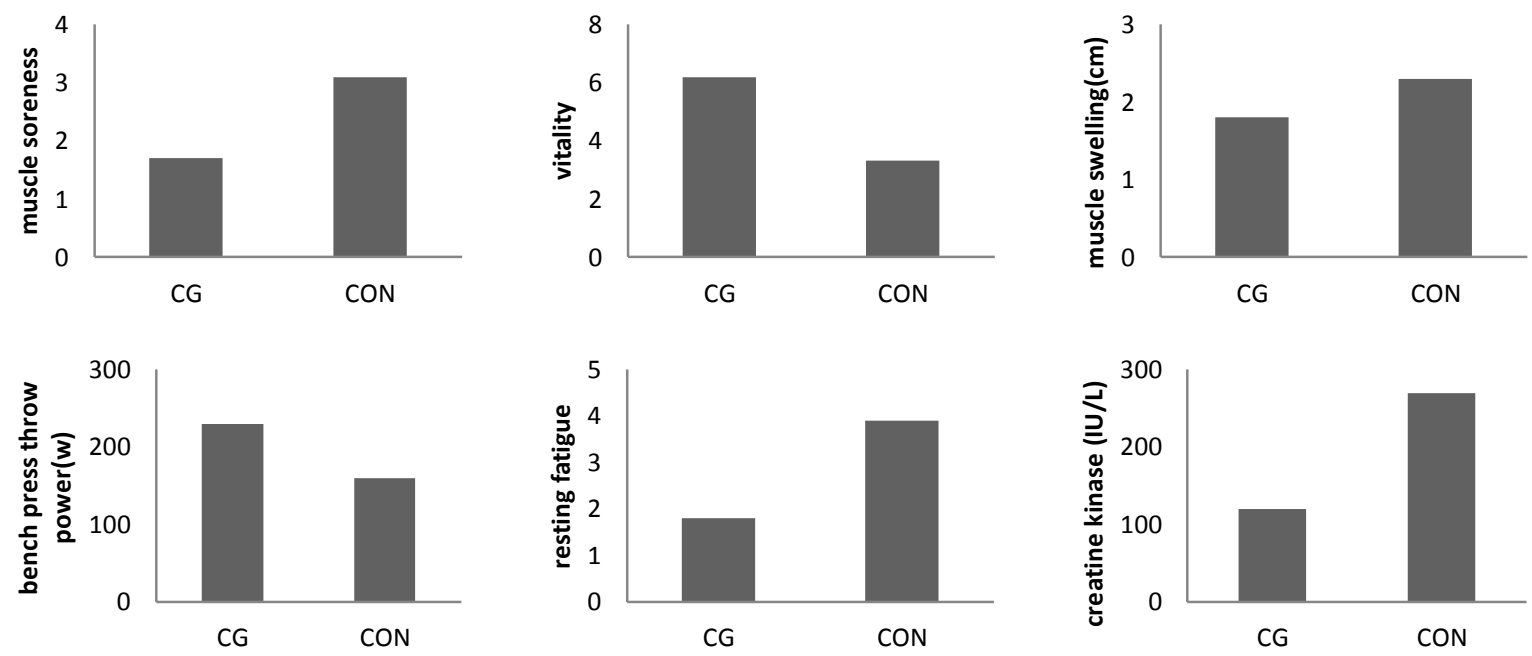

Figure 2. The mean $\pm \mathrm{SD}$ of the various parameters are presented for women comparing the compression garment (CG) with the control conditions(CON). $\mathrm{P} \leq 0.05$ from corresponding $\mathrm{CG}$ condition.

Interestingly, the bench throw performance was also significantly improved with the use of the whole body compression garment, reflecting enhanced recovery from the neuromuscular deficit created by the workout stress in the upper body.

Soreness ratings were significantly reduced in the upper torso when using the whole body compression garment. However, no changes were observed in the upper-body arm soreness levels. This might not be surprising with the use of resistance-trained subjects who have had extensive exposure to heavy eccentric loading $(34,35)$.

Although there were sex differences for magnitude of power production and also in the levels of muscle soreness and damage, a remarkable identical response profile of men and women was observed when comparing the whole body compression garment and control treatment conditions. It has been proposed that women may be protected in some ways by their sex from muscle damage because of estrogen biology, other hormonal and cytokine factors, and some inherent muscle/connective tissue characteristics in contraction dynamics (6). Interestingly, the same relative stress and response patterns for the different variables indicate that the workout was successful in producing an identical and relatively specific exercise stress in these resistance-trained men and women. Furthermore, the whole body garment construction was apparently successful in mediating the same successful recovery pattern for both men and women. 
Although no soreness ratings of the thighs were observed between the compression garment and control garment conditions, we did observe significantly lower ultrasound measures of muscle swelling in comparison with the control garment conditions. Prior research by Nosaka et al., 2002(33) demonstrated that soreness might not represent the amount of muscle tissue damage that might be present. Paradoxically, the performance variables involving the lower-body musculature were not affected by the workout to the extent observed in the upper body, but the ultrasound data demonstrate that tissue damage was present (31). Still, the compression effects of a lower amount of ultrasound-measured swelling was observed and demonstrated the effects of dynamic casting even in the thighs, which may also have contributed to the lower chemical damage markers as well (19). Additionally, lighter and moderate compression has also been shown to reduce venous blood pooling assisting the muscle pump in returning blood to the heart (37). A combination of such mechanisms might have been mediating mechanisms for faster lowerbody repair and performance maintenance. One salient feature that coincided with the reduction in overall general soreness/pain perceptions was the improvement in the vitality ratings along with the lower fatigue ratings with the use of the whole body compression garments. Lower perceived soreness ratings have been a prominent finding for the use of compression garments during a recovery period after an exercise stress $(7,9)$. The feelings of reduced fatigue and vitality may well have been because of the garment removal after 24 hours but might also reflect the gestalt of lower amounts of soft tissue damage as well. Nevertheless, such feelings are important for exercise readiness (23). It had been previously noted in extreme damage repair conditions, and use of a compressive sleeve resulted in greater discomfort over the first 24 hours and it produced a dramatic reduction in edema because of water being pushed out of the tissue biocompartment into the circulatory biocompartment via the compressive mechanical blocking (20). Such a perceptual response was not observed in this investigation because reductions in soreness, improved vitality, and reduced fatigue ratings were observed within 24 hours, indicating more rapid recovery responses than with severe soft tissue damage conditions. Thus, extreme soft tissue damage might produce higher levels of discomfort when the compression prevents muscle expansion, which was not observed in the limbs with this exercise stress. Recovery from a typical conditioning training session in trained men and women was ultimately enhanced with the use of a whole body compression garment.

We have recently shown that the magnetic resonance images of damage and CK markers responses of muscle damage followed a similar pattern (24). Clinical evaluation of muscle tissue damage was assessed biochemically with the standard measurements of total enzyme concentrations (i.e., $\mathrm{CK}$ and $\mathrm{LDH})$. Here, again the use of a whole body compression garment demonstrated significantly lower 24-hour CK and LDH concentrations compared with the control conditions. Control concentrations of these enzymes were well below high-damage or overtraining concentrations (13), marathon recovery values (29), or eccentric damage responses (32). Thus, the compression garment used reduced the level of CK and $\mathrm{LDH}$, indicating enhanced repair throughout the body's musculature even at lower levels of soft tissue damage. Davies et al., 2009(7) observed reductions in soreness at 48 hours after repetitive maximal drop jumps but no differences in CK concentrations comparing compression vs. control conditions. This may have been because of the rapid recovery of tissue for the specific exercise stress the athletes were exposed to similar to this study and effects were missed at the 48 -hour time point. Our findings of enhanced soft tissue repair with the use of compression might be well because of some limited immobilization (41) or more likely the limitation of extra movements with compression holding the limbs in what has been called "dynamic casting" effective in reducing soft tissue in damage repair studies (20). This might have also been an important factor in limiting the amount of excessive movement and impact oscillation of the body's musculature during normal activities and locomotion performed during the waking hours, which has been shown to be an important aspect of compression's mechanical characteristics at the compression levels used in the garments in this study (18). Interestingly, even with the use of the garment, sleep quality was not affected and if limbs were kept in a more elongated anatomical position, repair might have also been more geometrically optimized for repair. The practical applications of this study appear to demonstrate the efficacy of a whole body compression garment when recovery enhancement is needed after typical heavy resistance training. Reductions in muscle soreness and tissue damage appear to mediate enhanced performance and less fatigue and greater vitality. 
One can speculate that whole body compression garments used in this study may be useful in helping recovery with demanding tissue disruptive conditioning sessions or competitive events.

\section{ACKNOWLEDGMENTS}

We would like to thank a dedicated group of volunteers for this study, our laboratory support staff, our research assistants, and our physician and medical monitor, for his medical supervision of this study.

\section{REFERENCES}

1. Bahnert A, Norton K, Lock P. Association between post-game recovery protocols, physical and perceived recovery, and performance in elite Australian Football League players. J Sci Med Sport, 2013; 16:151-6.

2. Beaven CM, Maulder P, Pooley A, Kilduff L, Cook C. Effects of caffeine and carbohydrate mouth rinses on repeated sprint performance. Appl Physiol Nutr Metab, 2013; 38:633-7.

3. Bringard, A, Perrey, S, and Belluye, N. Aerobic energy cost and sensation responses during submaximal running exercise-Positive effects of wearing compression tights. Int J Sports Med, 2006; 27: 373-378.

4. Burden RJ, Glaister M. The effects of ionized and nonionized compression garments on sprint and endurance cycling. J Strength Cond Res, 2012; 26(10):2837-43.

5. Buysse, DJRI, CF, Monk, TH, Berman, SR, and Kupfer, DJ. Modified From: Rush J, et al, 2000: Handbook of Psychiatric Measures, APA, Pittsburgh Sleep Quality Index (PSQI). Washington DC.

6. Clarkson, PM and Hubal, MJ. Are women less susceptible to exercise-induced muscle damage? Curr Opin Clin Nutr Metab Care, 2001; 4: 527-531.

7. Davies, V, Thompson, KG, and Cooper, SM. The effects of compression garments on recovery. J Strength Cond Res, 2009; 23: 1786-1794.

8. Duffield R, Cannon J, King M. The effects of compression garments on recovery of muscle performance following highintensity sprint and plyometric exercise. J Sci Med Sport, 2010; $13: 136-40$

9. Duffield, R and Portus, M. Comparison of three types of fullbody compression garments on throwing and repeat-sprint performance in cricket players. Br J Sports Med, 2007; 41: 409-414; discussion 414.

10. Faude, O, Meyer, T, Urhausen, A, and Kindermann, W. Recovery training in cyclists: Ergometric, hormonal and psychometric findings. Scand J Med Sci Sports, 2009; 19: 433441.

11. Faulkner JA, Gleadon D, McLaren J, Jakeman JR. Effect of lowerlimb compression clothing on 400-m sprint performance. J Strength Cond Res, 2013 27:669-76.

12. Fleck SJ, Kraemer WJ. Designing Resistance Training Programs (3rd ed.). Champaign, IL: Human Kinetics Publishers, 2004.

13. Fry AC, Kraemer WJ, van Borselen F, Lynch JM, Marsit JL, Roy EP, Triplett NT, Knuttgen HG. Performance decrements with high-intensity resistance exercise overtraining. Med Sci Sports Exerc, 1994; 26: 1165-1173.

14. Gill ND, Beaven CM, Cook C. Effectiveness of post-match recovery strategies in rugby players. Br J Sports Med, 2006; 40:260-3.

15. Jakeman JR, Byrne C, Eston RG. Lower limb compression garment improves recovery from exercise-induced muscle damage in young, active females. Eur J Appl Physiol, 2010; 109:1137-44.

16. Judelson DA, Maresh CM, Farrell MJ, Yamamoto LM, Armstrong LE, Kraemer WJ, Volek JS, Spiering BA, Casa DJ, Anderson JM. Effect of hydration state on strength, power, and resistance exercise performance. Med Sci Sports Exerc, 2007; 39: 1817-1824.

17. Kraemer WJ, Bush JA, Bauer JA, Triplett-McBride NT, Paxton NJ, Clemson A, Koziris LP, Mangino LC, Fry AC, Newton RU. Influence of compression garments on vertical jump performance in NCAA Division I volleyball players. J Strength Cond Res, 1996; 10: 180-183.

18. Kraemer WJ, Bush JA, Newton RU, Duncan ND, Volek JS, Denegar CR, Canavan P, Johnston J, Putukian M, Sebastianelli WJ. Influence of a compressive garment on repetitive power output production before and after different types of muscle fatigue. Sports Med Train Rehabil, 1998; 8: 163-184.

19. Kraemer WJ, Bush JA, Wickham RB, Denegar CR, Go' mez AL, Gotshalk LA, Duncan ND, Volek JS, Newton RU, Putukian M, Sebastianelli WJ. Continuous compression as an effective therapeutic intervention in treating eccentric exercise-induced muscle soreness. Sport Rehabil, 2001; 10: 1123.

20. Kraemer WJ, Bush JA, Wickham RB, Denegar CR, Gomez AL, Gotshalk LA, Duncan ND, Volek JS, Putukian M, Sebastianelli WJ. Influence of compression therapy on symptoms following soft tissue injury from maximal eccentric exercise. J Orthop Sports Phys Ther, 2001; 31: 282-290.

21. Kraemer WJ, Dziados JE, Marchitelli LJ, Gordon SE, Harman EA, Mello R, Fleck SJ, Frykman PN, Triplett NT. Effects of different heavy-resistance exercise protocols on plasma betaendorphin concentrations. J Appl Physiol, 1993; 74: 450459.

22. Kraemer WJ, Fleck SJ, Dziados JE, Harman EA, Marchitelli LJ, Gordon SE, Mello R, Frykman PN, Koziris LP, Triplett NT. Changes in hormonal concentrations after different heavyresistance exercise protocols in women. J Appl Physiol, 1993; 75: 594-604.

23. Kraemer WJ, Hatfield DL, Spiering BA, Vingren JL, Fragala MS, Ho JY, Volek JS, Anderson JM, Maresh CM. Effects of a multinutrient supplement on exercise performance and hormonal responses to resistance exercise. Eur J Appl Physiol, 2007; 101: 637-646.

24. Kraemer WJ, Hatfield DL, Volek JS, Fragala MS, Vingren JL, Anderson JM, Spiering BA, Thomas GA, Ho JY, Quann EE, Izquierdo M, Hakkinen $\mathrm{K}$, Maresh CM. Effects of amino acids supplement on physiological adaptations to resistance training. Med Sci Sports Exerc, 2009; 41: 1111-1121.

25. Kraemer WJ, Joseph MF, Volek JS, Hoffman JR, Ratamess NA, Newton RU, Fragala MS, French DN, Rubin MA, Scheett TP, McGuigan MR, Thomas GA, Gomez AL, Hakkinen K, Maresh $\mathrm{CM}$. Endogenous opioid peptide responses to opioid andanti- 
inflammatory medications following eccentric exerciseinduced muscle damage. Peptides, 2009.

26. Kraemer WJ, Marchitelli L, Gordon SE, Harman E, Dziados JE, Mello R, Frykman P, McCurry D, Fleck SJ. Hormonal and growth factor responses to heavy resistance exercise protocols. J Appl Physiol, 1990; 69: 1442-1450.

27. Kraemer WJ, Volek JS, Bush JA, Gotshalk LA, Wagner PR, Gomez AL, Zatsiorsky VM, Duarte M, Ratamess NA Mazzetti SA, Selle BJ. Influence of compression hosiery on physiological responses to standing fatigue in women. Med Sci Sports Exerc, 2000; 32: 1849-1858.

28. MacRae BA, Cotter JD, Laing RM. Compression garments and exercise: garment considerations, physiology and performance. Sports Med, 2011; 41:815-43.

29. Maresh CM, Allison TG, Noble BJ, Drash A, Kraemer WJ. Substrate and hormone responses to exercise following a marathon run. Int J Sports Med, 1989; 10: 101-106.

30. Marshall HS, Gold MS, Gent R, Quinn PJ, Piotto L, Clarke MF, Roberton DM. Ultrasound examination of extensive limb swelling reactions after diphtheria-tetanus-acellular pertussis or reduced-antigen content diphtheria-tetanus-acellular pertussis immunization in preschool-aged children. Pediatrics, 2006; 118: 1501-1509.

31. Noriyuki A, Kotaro Y, Takafumi K, Gojiro N, Shinji I, Takuya H, Jun A, Isao K, Hiromi S. Ultrasound Assessment of deep tissue injury in pressure ulcers: Possible prediction of pressure ulcer progression. Plastic and Reconstructive Surgery, 2009; 124: 540-550.

32. Nosaka K, Clarkson PM. Variability in serum creatine kinase response after eccentric exercise of the elbow flexors. Int J Sports Med, 1996; 17: 120-127.
33. Nosaka K, Newton, M. Repeated eccentric exercise bouts do not exacerbate muscle damage and repair. J Strength Cond Res, 2002; 16: 117- 122.

34. Nosaka K, Newton M, Sacco P. Delayed-onset muscle soreness does not reflect the magnitude of eccentric exerciseinduced muscle damage. Scand J Med Sci Sports, 2002; 12: 337-346.

35. Nosaka K, Sakamoto K, Newton M, Sacco P. The repeated bout effect of reduced-load eccentric exercise on elbow flexor muscle damage. Eur J Appl Physiol, 2001; 85: 34-40.

36. Pearce AJ, Kidgell DJ, Grikepelis LA, Carlson JS. Wearing a sports compression garment on the performance of visuomotor tracking following eccentric exercise: A pilot study. J Sci Med Sport, 2009; 12: 500-502.

37. Platts SH, Tuxhorn JA, Ribeiro LC, Stenger MB, Lee SM, Meck JV. Compression garments as countermeasures to orthostatic intolerance. Aviat Space Environ Med, 2009; 80: 437-442.

38. Scanlan AT, Dascombe BJ, Reaburn PR, Osborne M. The effects of wearing lower-body compression garments during endurance cycling. Int J Sports Physiol Perform, 2008; 3:42438 .

39. Silver T, Fortenbaugh D, Williams R. Effects of the bench shirt on sagittal bar path. J Strength Cond Res, 2009; 23: 1125-1128.

40. Yeargin SW, Casa DJ, Armstrong LE, Watson G, Judelson DA, Psathas E, Sparrow, SL. Heat acclimatization and hydration status of American football players during initial summer workouts. J Strength Cond Res, 2006; 20: 463-470.

41. Zainuddin Z, Hope P, Newton M, Sacco P, Nosaka K. Effects of partial immobilization after eccentric exercise on recovery from muscle damage. J Athletic Train, 2005; 40: 197-202. 